\title{
Platelet to Lymphocyte Ratio as a Predictor of Infarct-Related Artery Patency in Patients Undergoing Primary Percutaneous Coronary Intervention
}

\author{
Abdalla Mostafa Kamal ${ }^{1}$, Mona Fikry Fathy ${ }^{2}$, Ghada Mahmoud Soltan ${ }^{1}$ \\ ${ }^{1}$ Cardiology Department, Faculty of Medicine, Menofia University, Al Minufya, Egypt \\ ${ }^{2}$ Cardiology Department, National Heart Institute, Cairo, Egypt
}

Email address:

monadrcardio@gmail.com (M. F. Fathy)

\section{To cite this article:}

Abdalla Mostafa Kamal, Mona Fikry Fathy, Ghada Mahmoud Soltan. Platelet to Lymphocyte Ratio as a Predictor of Infarct-Related Artery Patency in Patients Undergoing Primary Percutaneous Coronary Intervention. Cardiology and Cardiovascular Research.

Vol. 5, No. 2, 2021, pp. 61-66. doi: 10.11648/j.ccr.20210502.12

Received: April 15, 2021; Accepted: May 3, 2021; Published: May 14, 2021

\begin{abstract}
Background: In myocardial infarction (MI) patients, myocardial reperfusion and subsequently, early infarct related artery (IRA) patency are very critical in this emergency situation. Recently, there is growing research on the ability of platelet to lymphocyte ratio (PLR) to be a predictor of outcome and its value as a marker for inflammation and coagulopathy detection. Our work aims at finding the relation between PLR and the prognosis of IRA in STEMI patients. Methodology: Two hundred cases presented with STEMI were included for the study. Patency of IRA was estimated by Thrombolysis in Myocardial Infarction (TIMI) grade. Patent IRA was defined as TIMI grade III flow and occluded IRA was defined as TIMI grade 0-II flow. Blood samples were withdrawn on admission at emergency department to calculate PLR. Results: Forty-one (20.5\%) cases revealed TIMI 3 flow in IRA before pPCI. Occluded group showed significantly higher PLR than patent group with mean PLR $231.3 \pm 94.2$ vs $100.95 \pm 37.7$ respectively with $\mathrm{P}$ value $<0.0001$. Multivariate regression analysis demonstrated, both HTN [95\% CI (- 0.135)-(-0.747)] \& PLR ratio [95\% CI (-0.001)-(-0.002)] together are the most independent predictors for TIMI flow in IRA (F-ratio=12.2, $\mathrm{p}<0.001)$. Conclusion: our results show that high Platelet lymphocyte ratio predicts patency of IRA independently in cases with STEMI before pPCI.
\end{abstract}

Keywords: PLR, IRA, pPCI

\section{Introduction}

The pathological process of coronary atherosclerosis leads eventually to thrombosis and occlusion of coronary arteries resulting in cardiac muscle ischemia and can be sever to level of muscle tissue ischemic necrosis or what is called myocardial infarction (MI) and in acute early cases it lead to electrocardiography heart changes and is termed ST-segment elevation myocardial infarction (STEMI) $[1,2]$

To achieve IRA patency, Primary percutaneous coronary intervention (pPCI) is a standard of care and it is urged to be done as early as possible to prevent $\mathrm{MI}$ and improve outcomes [3], It has been agreed that the prognosis and cardiac muscle recovery in STEMI cases after pPCI is affected by the IRA patency before pPCI [4].

The Inflammatory process plays a pivotal role in atherosclerosis formation and its establishment [5, 6]. Total leucocyte count (TLC) has been studied as a marker for inflammation and whether it has a prognostic value in coronary artery atherosclerosis $[7,8]$.

Thrombocytes have central role in the mechanism of thrombosis of atherosclerosed arteries and increased count of platelets is correlated with worse prognosis of MI [9, 10].

Platelet lymphocyte ratio (PLR) is an index that can be used as a marker of ongoing inflammatory process which depends on platelet count and lymphocyte count at the same time $[11,12]$.

Moreover, PLR has been studied in cases with advanced coronary disease reflected in the higher Syntax scores and no-reflow phenomenon and it was found that PLR is an independent risk factor, [13] However, the research about the prognostic significance of IRA patency before pPCI in 
STEMI cases is lacking, which make it an attractive investigational subject.

\section{Patients and Methods}

\subsection{Study Design}

Our study was a cross-sectional observational study conducted at two tertiary referral centers on cases presented with STEMI and undergoing pPCI admitted at National heart institute and Menofia university hospital between January 2019 and March 2020. A total of 200 cases were enrolled in the study and divided into occluded group and patent group according to TIMI flow grade in IRA.

\subsection{Methods}

Patients with former attack of MI, Valvular dysfunction, Presence of kidney or liver disease, Presence of Focus of infection, Malignancy, Autoimmune or hematologic disease and/or Chronic obstructive lung disease were excluded from the study All cases were reviewed for their demographics, clinical characteristics and risk profile. Twelve leads electrocardiography (ECG) on admission and post PCI conventional echocardiography for evaluation of left ventricular (LV) function using ejection fraction $(\mathrm{EF} \%)$ were performed. Laboratory investigations including Complete blood count (CBC) from which platelet count, lymphocyte count and platelet to lymphocyte ratio were obtained, serum creatinine and cardiac biomarkers. Labelled samples were obtained from venous blood of cases and transferred immediately to the emergency laboratory for immediate counting. Ejection fraction (EF) was estimated by transthoracic echocardiography using modified simpson after PCI immediately [14]. Coronary angiogram (CA) and (TIMI) scale were done by two study blinded interventional cardiologists. CA was done in two planes and according to Judkins method. TIMI flow grading was done before pPCI for every patient. TIMI grade 0, I, and II were categorized as non-patent flow. Patent flow was given TIMI grade III [15-17].

\section{Statistics}

Data was entered into the Statistical Package for the Social Science (SPSS). Results were represented as median and percentiles for non-parametric quantitative measures. Numbers and percentage were used for categorized data. Categorical variables were compared with chi-square test, and continuous variables were compared with Wilcoxon Rank Sum test or Kruskall Wallis test. Multivariate, stepwise backward conditional logistic regression analysis was used to detect the independent predictors of IRA patency, and all significant parameters in the univariate analysis were selected in the multivariate model.. A cutoff value for PLR to predict patency of IRA was evaluated using receiveroperating characteristics (ROC) curve analysis. The confidence interval (CI) was set to $95 \%$ and the margin of error accepted was set to $5 \% . \quad \mathrm{P} \leq 0.05$ was considered statistically significant.

\section{Results}

Angiographic data and clinical data of 200 cases were included in the final study. Results showed that One hundred sixty-two $(81 \%)$ cases were male and thirty nine $(19 \%)$ cases were female, their mean age was 56.2 years \pm 9.48 . Out of all the cases One hundred-fourteen $(57.3 \%)$ were smokers, sixtyfour $(32 \%)$ were diabetics and ninety- three (46.5\%) were hypertensive. The infarction site was found to be anterior STEMI in one hundred-seventeen $(58.5 \%)$ cases, inferior STEMI in seventy-three $(36.5 \%)$ cases and lateral STEMI in ten $(5 \%)$ cases. Twenty-two (11\%) cases had no risk factors, ninety-five $(47.5 \%)$ cases had one risk factor, seventy-three $(36.5 \%)$ cases had two risk factors while ten $(5 \%)$ cases had three risk factors. The mean serum creatinine after PCI was $1.1 \pm 0.31$, mean left ventricular ejection fraction (LVEF) was $47.89 \% \pm 10.35$ and mean PLR was $198.9 \pm 90.22$. Patient's characteristics and baseline laboratory parameters are demonstrated in table 1 according to TIMI grade.

Table 1. Patient characteristics.

\begin{tabular}{|c|c|c|c|}
\hline & Occluded group $(n=159)$ & Patent Group $(n=41)$ & $P$ value \\
\hline Age & $56.20 \pm 9.480$ & $54.7 \pm 11.59$ & $>0.05$ \\
\hline Male Gender (\%) & $129(81.1 \%)$ & $33(80.4 \%)$ & $>0.05$ \\
\hline Diabetic $(\%)$ & $53(33.3 \%)$ & $11(26.8 \%)$ & $>0.05$ \\
\hline HTN (\%) & $82(51.5 \%)$ & $11(26.8 \%)$ & $<0.05$ \\
\hline Smokers (\%) & $89(55.9 \%)$ & $25(60.9 \%)$ & $>0.05$ \\
\hline \multicolumn{4}{|l|}{ Site of Infarction } \\
\hline ant.STEMI & $93(58.4 \%)$ & $24(58.5 \%)$ & $>0.05$ \\
\hline Inf STEMI & $57(35.8 \%)$ & $16(39 \%)$ & $>0.05$ \\
\hline LVEF (\%) & $47.9 \% \pm 10.4$ & $58.4 \% \pm 6.8$ & $<0.0001$ \\
\hline S.creatinine & $1.01 \pm 0.31$ & $0.99 \pm 0.53$ & $>0.05$ \\
\hline Platelet $\left(\times 10^{3} / \mu \mathrm{L}\right)$ & $279 \pm 55.23$ & $199.6 \pm 46.5$ & $<0.0001$ \\
\hline Lymphocyte $\left(\mathrm{x} 10^{3} / \mu \mathrm{L}\right)$ & $1.38 \pm 0.52$ & $2.1 \pm 0.5$ & $<0.0001$ \\
\hline PLR & $231.3 \pm 94.2$ & $100.95 \pm 37.7$ & $<0.0001$ \\
\hline
\end{tabular}

When Cases were categorized according to TIMI grade of IRA into patent group with TIMI III grade and occluded group with TIMI 0-II grade, results showed no clinical significance regarding age, gender, rate of DM, rate of smoking, site of infarction while rate of hypertension and post procedural (LVEF) showed significant difference 
between the two groups ( $p$ value $<0.05,<0.0001$ respectively). As regards to laboratory parameters; platelet count showed significantly higher results in occluded group while lymphocytic count showed significantly lower results in occluded group ( $p$ value $<0.0001$ ). At the same time, PLR had a significantly higher values in occluded group compared to patent group $(231.3 \pm 94.2$ vs $100.95 \pm 37.7$, p value $<0.0001)$.
Coronary angiograph was done and results showed that One hundred (50\%) cases showed TIMI 0 grade in IRA while TIMI I, TIMI II and TIMI III grade of IRA was shown in thirty cases $(15 \%)$, Twenty nine cases $(14.5 \%)$ and Forty-one (20.5\%) cases respectively. Associated other non culprit vessel disease was found in Ninety-two (46\%) cases. Angiographic data according to TIMI grade are represented in table 2.

Table 2. Angiographic data.

\begin{tabular}{lll}
\hline & TIMI 0-II $(\mathbf{n = 1 5 9 )}, \mathbf{7 9 . 5 \%}$ & TIMI III (n=41), 20.5\% \\
\hline Site of infarct related artery & & P value \\
LAD & $95(59.70 \%)$ & $24(58.5 \%)$ \\
LCX & $19(11.9 \%)$ & $1(2.4 \%)$ \\
RCA & $36(22.6 \%)$ & $16(39.1 \%)$ \\
OM & $9(5.6 \%)$ & 0 \\
Non culprit Vessels affected & $1.70 \pm 0.861$ & $1.54 \pm 0.745$ \\
\hline
\end{tabular}

Non significant results were found between occluded and patent groups as regards to site of IRA with p value $>0.05$. Similarly, no significance was found regarding number of non culprit vessels affected in occluded group vs patent group ( $1.70 \pm 0.861$ vs $1.54 \pm 0.745$ respectively, $\mathrm{p}$ value $>0.05)$

Moreover, a correlation between TIMI flow in IRA to all risk factors and angiographic data was done. Results are shown in table 3 .

Table 3. Relation of TIMI flow to clinical variables.

\begin{tabular}{|c|c|c|c|c|c|}
\hline & TIMI 0 & TIMI I/II & TIMI III & $P$ value & \\
\hline (No) of patients & 100 & 59 & 41 & & \\
\hline \multirow{2}{*}{ Male $(\%)$} & 82 & 47 & 33 & & \\
\hline & $82 \%$ & $79.60 \%$ & $80.4 \%$ & & \\
\hline \multirow{2}{*}{ Female $(\%)$} & 18 & 12 & 8 & & \\
\hline & $18 \%$ & $20.3 \%$ & $19.5 \%$ & & \\
\hline \multirow{2}{*}{ Diabetic (\%) } & 29 & 24 & 11 & \multirow{2}{*}{$>0.05$} & \multirow{2}{*}{ NS } \\
\hline & $29 \%$ & $40.6 \%$ & $26.8 \%$ & & \\
\hline HTN & 50 & 32 & 11 & \multirow{2}{*}{$<0.05$} & \multirow{2}{*}{$\mathrm{S}$} \\
\hline$(\%)$ & $50 \%$ & $54.2 \%$ & $26.8 \%$ & & \\
\hline \multirow{2}{*}{ Smokers (\%) } & 57 & 32 & 25 & \multirow{2}{*}{$>0.05$} & \multirow{2}{*}{ NS } \\
\hline & $57 \%$ & $54.2 \%$ & $60.9 \%$ & & \\
\hline \multirow{2}{*}{ Anterior STEMI } & 58 & 35 & 24 & \multirow{2}{*}{$>0.05$} & \multirow{2}{*}{ NS } \\
\hline & $58 \%$ & $59.3 \%$ & $58.5 \%$ & & \\
\hline \multirow{2}{*}{ Lateral STEMI } & 5 & 4 & 1 & \multirow{2}{*}{$>0.05$} & \multirow{2}{*}{ NS } \\
\hline & $5 \%$ & $6.7 \%$ & $2.43 \%$ & & \\
\hline \multirow{2}{*}{ Inferior STEMI } & 37 & 20 & 16 & \multirow{2}{*}{$>0.05$} & \multirow{2}{*}{ NS } \\
\hline & $37 \%$ & $33.8 \%$ & $39.0 \%$ & & \\
\hline \multirow{2}{*}{ LAD } & 60 & 35 & 24 & \multirow{2}{*}{$>0.05$} & \multirow{2}{*}{ NS } \\
\hline & $60 \%$ & $59.3 \%$ & $58.5 \%$ & & \\
\hline \multirow{2}{*}{ LCX } & 13 & 6 & 1 & \multirow{2}{*}{$>0.05$} & \multirow{2}{*}{ NS } \\
\hline & $13 \%$ & $10.1 \%$ & $2.4 \%$ & & \\
\hline \multirow{2}{*}{ RCA } & 19 & 17 & 16 & \multirow{2}{*}{$>0.05$} & \multirow{2}{*}{ NS } \\
\hline & $19 \%$ & $28.8 \%$ & $39.0 \%$ & & \\
\hline \multirow{2}{*}{ Om } & 8 & 1 & 0 & \multirow{2}{*}{$>0.05$} & \multirow{2}{*}{ NS } \\
\hline & $8 \%$ & $1.60 \%$ & $0.00 \%$ & & \\
\hline
\end{tabular}

Among all variables only presence of hypertension as a risk factor was significantly prevalent in patient with non patent IRA with $\mathrm{p}$ value $<0.05$.

In correlation analysis between PLR to Risk factors and angiographic data a significant correlation was found between PLR and TIMI flow in IRA with P value $<0.0001$. Figure 1 illustrates the correlation between PLR and TIMI flow in IRA.

In the multiple logistic regression analysis, both HTN $[95 \%$ CI (- 0.135)-(-0.747)] \& PLR ratio [95\% CI (-0.001)-(-0.002)] together are the most independent predictors for TIMI (Fratio $=12.2, \mathrm{p}<0.001$ ). Results are shown in table 4 .
Table 4. Predictors of patency of IRA.

\begin{tabular}{lllll}
\hline Item & Reg. Coef. & t & P & Sig. \\
\hline (Constant) & 2.173 & 3.56 & 0 & HS \\
Age & -0.008 & -0.895 & 0.372 & NS \\
Gender & -0.135 & -0.547 & 0.585 & NS \\
DM & -0.002 & -0.009 & 0.992 & NS \\
HTN & -0.483 & -2.836 & 0.005 & HS \\
Smk & -0.145 & -0.704 & 0.482 & NS \\
P_L & -0.002 & -4.297 & 0 & HS \\
\hline
\end{tabular}


Using a PLR cutoff value of 109.4 , PLR predicted nonpatent flow (no-reflow) with $65.9 \%$ sensitivity and $89.9 \%$ specificity (AUC: 90.3\%; 95\% CI: 86.1-94.5). Figure 2 illustrates ROC curve analysis of PLR.

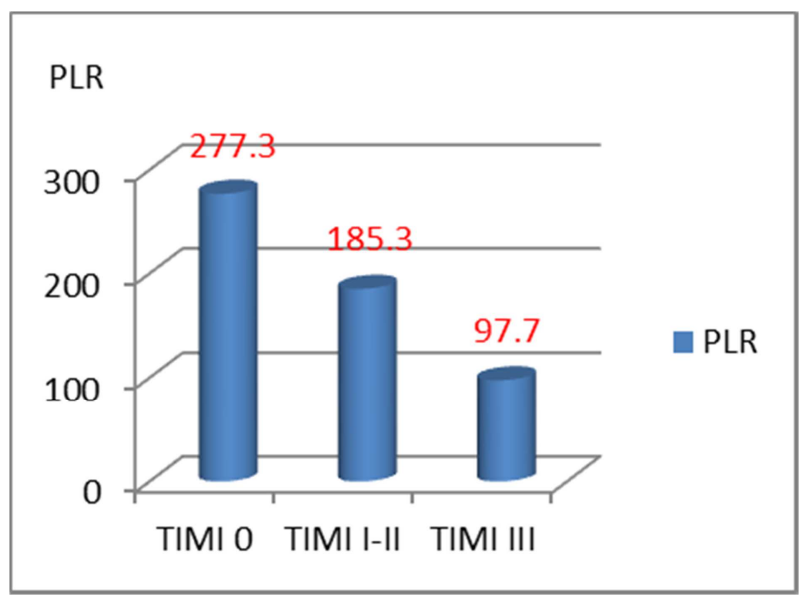

Figure 1. Relation between PLR and TIMI flow.

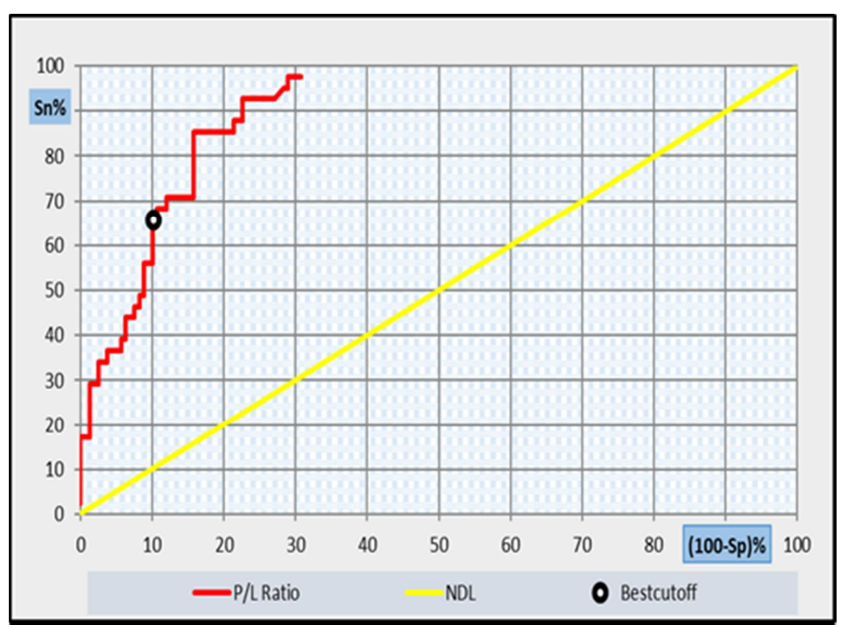

Figure 2. ROC curve analysis showing the diagnostic performance of $P / L$ Ratio for discriminating TIMI 0, 1, 2 Vs 3 [AUC: PLR 0. 903 95\% CI (86.194.5)].

\section{Discussion}

Our study shows that PLR was an independent predictor of patency of IRA in cases with STEMI undergoing pPCI. PLR was directly correlated to number of risk factors and showed higher values in hypertensive cases. In this study cases who were presented with STEMI and had normal coronary flow (patent group with TIMI III) in IRA before PCI had significantly lower platelet count and PLR in comparison to cases with occluded IRA (TIMI 0-II).

IRA is crucial for the prognosis of cases with STEMI. Cases with pre-PCI normal flow of IRA have higher postPCI TIMI grades [18], decreased rates of heart failure and cardiogenic shock, improved early and late LVEFs, and reduced short- and long-term mortality [19].

Different biomarkers have been studied as predictors of IRA. Şahin et al [20] assessed uric acid (UA) and neuterophil to lymphocytes ratio (NLR) as possible predictors of IRA in cases with STEMI before pPCI. Moreover, Li et al [21] screened the independent predictors of spontaneous reperfusion (SR) in IRA defined as TIMI III grade before pPCI. In our study we assessed the possibility of PLR to predict patency of IRA in STEMI before pPCI.

Recently, PLR, has been proposed to be a pro-thrombotic and inflammatory marker [11, 22, 23], our findings confirm the relation between these markers and patient risk beforeprimary PCI.

Coronary artery occlusion begins with erosion of atherosclerotic plaques that accumulate and stop flow of the blood in the coronary artery leading to inhibiting blood supply to cardiac muscle. However, the mechanism of initiation of atherosclerotic plaque erosion is still unclear. Inflammation has shown its powerful effect both in initiation and in progression of this process, besides it has an active role during the process of atherosclerosis [24].

Many studies adopted assessment of PLR as biomarker in cardiovascular diseases for its low cost and availability. Bekler et al [25] evaluated PLR as a predictor for left ventricular systolic dysfunction (LVSD) in cases with non-ST elevated acute coronary syndrome (NST-ACS) and results showed that PLR was significantly higher [164.2 (66.3$724.3)$ vs. 101.5 (20.7-334.4), $\mathrm{p}<0.001]$ in cases with LVSD. PLR was negatively correlated with LVEF in the correlation analysis $(\mathrm{r}=-\mathrm{0}=400, \mathrm{p}<0.001)$. In our study PLR was negatively correlated to LVEF in the correlation analysis $(\mathrm{r}=$ $0.316, \mathrm{p}<0.001)$ in cases with ST elevated acute coronary syndrome (STEMI-ACS).

Acet et al [26] studied the relation between PLR, NLR and UA to patency of IRA before pPCI. Cases were categorized according to TIMI grade of IRA before pPCI. Cases with a TIMI grade III were placed into the spontaneous reperfusion (SR) group, while cases with TIMI grade of 0 -II were placed into the group of non spontaneous reperfusion ( non-SR group). Both groups were found to be identical regarding risk factors for atherosclerosis, systolic blood pressure measurements and heart rate. LVEF was significantly lower in the Non-SR group than the SR-group $(42.4 \pm 10.2 \%$ versus $46.2 \pm 9.5 \%$, respectively; $p=0.003)$. SR group had significantly lower UA, PLR and NLR than in the Non-SR group. PLR with 190 cutoff value predicted impaired IRA TIMI flow grade before primary PCI with a sensitivity of $87.5 \%$ and a specificity of $83.5 \%$ [ROC (AUC) $0.603,95 \%$ CI $0.538-0.669, \mathrm{p}=0.004]$.

In our study we investigated the association of PLR only to the patency of IRA before pPCI. Our study was similar to the previous mentioned study in that we found that LVEF was significantly lower in occluded group than patent group $[47.8 \% \pm 10.3 \%$ vs $58.4 \% \pm 6.9 \%$ respectively, $(\mathrm{p}$ value $<0.001)]$ and that PLR is an independent predictor of patency in IRA before pPCI $(\mathrm{F}-\mathrm{ratio}=12.2, \mathrm{p}<0.001)$. However it differs in that our results found that there was significant difference between the two groups regarding presence of Hypertension as a CVD risk factor $(p<0.05)$ and the best cut off value for PLR to discriminate between 
patent and non patent IRA is at 109.4; with specificity $89.9 \%$; sensitivity $65.9 \%$.

Yayla et al [27] showed that, on admission PLR had 71.5\% sensitivity and $60 \%$ specificity in predicting non-patent flow (no-reflow) in IRA with a PLR cutoff value of 80.2. Patency of IRA was evaluated in 452 cases by (TIMI) grade. Before pPCI TIMI grade III flow in IRA was in 92 (20.4\%) cases. The occluded IRA group showed significantly higher PLR $(138.4+51.4$ vs $95.4+43.5, \mathrm{P}<.001)$ and also significantly higher NLR ( $\mathrm{P}<.05)$. Multivariate regression analysis demonstrated the PLR ([OR]: 0.987; 95\%CI: 0.978-0.995, P 1/4.002) and NLR (OR: 0.758; 95\% CI: 0.584-0.985, P 1/4.038) on admission are independent predictors of IRA patency.

Regarding age, gender, and atherosclerosis risk factors, there was no statistically significant difference among the studied groups $(\mathrm{P}>.05)$. IRAs were also similar between the groups (P 1/4.261). In the occluded group, the majority $(84.7 \%)$ of cases revealed TIMI 0 flow in IRA.

In the current study we demonstrated that PLR is an important predictor of patency of IRA in cases presented with STEMI before pPCI, PLR was predominantly elevated in occluded IRA.

In the present study we enrolled 200 cases with STEMI who were treated with primary PCI, $79.5 \%$ showed non patent IRA in their CA before PPCI.

The present study demonstrated that high PLR is associated with a higher degree of obstruction of IRA and PLR value is a correlate of TIMI flow in IRA. We demonstrated that platelet count and PLR were significantly increased in occluded IRA than patent IRA group, $(279.03 \pm 55.2$ vs $199.6 \pm 46.5, \mathrm{p}$ value $<0.001$ and $231.3 \pm 94.2$ vs $100.9 \pm 97.7, \mathrm{p}$ value $<0.001$ respectively). In logistic regression analysis, PLR (95\%CI: $-0.002-0.001 \mathrm{p}<0.000)$ and HTN $(95 \% \mathrm{CI}:-0.747-0.135 \mathrm{p}=0.005)$ were independent predictors of patency in IRA. Diagnostic validity test showed that the best cutoff value for PLR to discriminate between patent and non patent IRA is at 109.4; with specificity $89.9 \%$; sensitivity $65.9 \%$; predictive negative; predictive positive and efficacy were; $91.1 ; 62.8 ; 85.0 \%$ respectively (AUC: $90.3 \%$; 95\% CI: 86.1-94.5).

In our study, results showed no clinical significance regarding age, gender, rate of DM, rate of smoking, site of infarction while rate of hypertension and post procedural LVEF showed significant difference between the two groups ( $p$ value $<0.05,<0.0001$ respectively)

In agreement with the recent published data, our study shows the important role of inflammatory markers and brings autoimmunity to the scope in the pathophysiological mechanism of the no-reflow phenomenon in IRA before pPCI. However, further research is needed to describe the exact role of PLR in the no-reflow incidence.

\section{Limitations}

There were limitations to this study. First, the study group was small. Second, we had not followed the cases for adverse cardiac event and explore the relation between these cardiac events and PLR. Finally, we did not estimate markers of inflammation including CRP and fibrinogen or other cytokines.

\section{Conclusion}

Our results demonstrate the presence of a significant correlation between PLR at presentation and the amount of myocardial blood flow before PPCI in cases with STEMI. The PLR was found to be an independent predictor of an occluded IRA in STEMI cases underwent pPCI. This proves that PLR is a convenient, easy, available and economical parameter, which can independently, predicts IRA patency in every day practice for cases with ischemic heart emergency.

\section{References}

[1] Maseri A, Fuster V. Is there a vulnerable plaque? Circulation. 2003; 107 (16): 2068-2071.

[2] Fuentes QE, Fuentes QF, Andres V, Pello OM, Font de Mora J, Palomo GI. Role of platelets as mediators that link inflammation and thrombosis in atherosclerosis. Platelets. 2013; 24 (4): 255-262.

[3] Zeymer U, Huber K, Fu Y, et al. Impact of TIMI 3 patency before primary percutaneous coronary intervention for STelevation myocardial infarction on clinical outcome: results from the ASSENT-4 PCI study. Eur Heart J Acute Cardiovasc Care. 2012; 1 (2): 136-42.

[4] Brodie BR, Stuckey TD, Hansen C, et al. Benefit of coronary reperfusion before intervention on outcomes after primary angioplasty for acute myocardial infarction. Am J Cardiol. 2000; 85 (1): 13-18.

[5] Ross R. Atherosclerosis: An inflammatory disease. N Engl J Med 1999; 340: 115-26.

[6] Libby P. What have we learned about the biology of atherosclerosis? The role of inflammation. Am J Cardiol 2001; 88: $3 \mathrm{~J}-6 \mathrm{~J}$.

[7] Gurm HS, Bhatt DL, Gupta R, Ellis SG, Topol EJ, Lauer MS. Preprocedural white blood cell count and death after percutaneous coronary intervention. Am Heart J 2003; 146: 692-8.

[8] Gillum RF, Mussolino ME, Madans JH. Counts of neutrophils, lymphocytes, and monocytes, cause-specific mortality and coronary heart disease: the NHANES-I epidemiologic followup study. Ann Epidemiol 2005; 15: 266-71.

[9] Klovaite J, Benn M, Yazdanyar S, Nordestgaard BG. High platelet volume and increased risk of myocardial infarction: 39,531 participants from the general population. J Thromb Haemost. 2011; 9 (1): 49-56.

[10] Ly HQ, Kirtane AJ, Murphy SA, et al. Association of platelet counts on presentation and clinical outcomes in ST-elevation myocardial infarction (from the TIMI Trials). Am J Cardiol. 2006; 98 (1): $1-5$

[11] Ommen SR, Hammill SC, Gibbons RJ. The relative lymphocyte count predicts death in patients receiving implantable cardioverter defibrillators. Pacing Clin Electrophysiol 2002; 25 (10): 1424-8. 
[12] Arruda-Olson AM, Reeder GS, Bell MR, Weston SA, Roger VL. Neutrophilia predicts death and heart failure after myocardial infarction: a community-based study. Circ Cardiovasc Qual Outcomes 2009; 2 (6): 656-62.

[13] Yildiz A, Yuksel M, Oylumlu M, Polat N, Akyuz A, Acet H, et al.. The Utility of the Platelet-Lymphocyte Ratio for Predicting No Reflow in Patients With ST-Segment Elevation Myocardial Infarction. Clin Appl Thromb Hemost 2014. http://dx.doi.org/10.1177/1076029613519851 [Epub ahead of print].

[14] Lang RM, Bierig M, Devereux RB, et al., Chamber Quantification Writing Group, American Society of Echocardiography's Guidelines and Standards Committee, European Association of Echocardiography, Recommendations for chamber quantification: a report from the American Society of Echocardiography's Guidelines and Standards Committee and the Chamber Quantification Writing Group, developed in conjunction with the European Association of Echocardiography, a branch of the European Society of Cardiology, J Am Soc Echocardiogr, 2005; 18 (12): 1440-63.

[15] Dogan M, Akyel A, Bilgin M, et al. Can admission neutrophil to lymphocyte ratio predict infarct-related artery patency in STSegment elevation myocardial infarction. Clin Appl Thromb Hemost. 2015; 21 (2): 172-176.

[16] Nunez J, Nunez E, Bodi V, et al. Usefulness of the neutrophil to lymphocyte ratio in predicting long-term mortality in ST segment eleation myocardial infarction. Am J Cardiol. 2008; $101(6): 7$.

[17] Lamas GA, Flaker GC, Mitchell G, et al. Effect of infarct artery patency on prognosis after acute myocardial infarction. The Survival and Ventricular Enlargement Investigators. Circulation. 1995; 92 (5): 1101-1109.

[18] Mehta RH, Harjai KJ, Cox D, et al. Clinical and angiographic correlates and outcomes of suboptimal coronary flow inpatients with acute myocardial infarction undergoing primary percutaneous coronary intervention. J Am Coll Cardiol. 2003; 42 (10): 1739-1746.

[19] Stone GW, Cox D, Garcia E, Brodie BR, Morice MC, Griffin $\mathrm{J}$, et al. Normal flow (TIMI-3) before mechanical reperfusion therapy is an independent determinant of survival in acute myocardial infarction: analysis from the primary angioplasty in myocardial infarction trials. Circulation 2001; 104: 636-41.

[20] D Yıldıray Şahin, M Gür, Z Elbasan; et al. Predictors of preinterventional patency of infarct-related artery in patients with ST-segment elevation myocardial infarction: Importance of neutrophil to lymphocyte ratio and uric acid level. Exp Clin Cardiol 2013; 18 (2): e77-e81.

[21] Jing Li, Ying Zhou, Yaowen Zhang et al. Admission homocysteine is an independent predictor of spontaneous reperfusion and early infarct-related artery patency before primary percutaneous coronary intervention in ST-segment elevation myocardial infarction. BMC Cardiovascular Disorders (2018) 18: 125.

[22] Smith RA, Ghaneh P, Sutton R, Raraty M, Campbell F, Neoptolemos JP. Prognosis of Resected Ampullary Adenocarcinoma by Preoperative Serum CA19-9 Levels and Platelet-Lymphocyte Ratio. J Gastrointest Surg 2008; 12: 1422-8.

[23] Wang D, Yang JX, Cao DY, et al. Preoperative neutrophillymphocyte and plateletlymphocyte ratios as independent predictors of cervical stromal involvement in surgically treated endometrioid adenocarcinoma. Onco Targets Ther. 2013; 6: 211-6.

[24] Libby P, Ridker PM, Maseri A. Inflammation and atherosclerosis. Circulation. 2002; 105 (9): 1135-1143.

[25] Bekler A, Gazi E, Yilmaz M et al. Could elevated plateletlymphocyte ratio predict left ventricular systolic dysfunction in patients with non-ST elevated acute coronary syndrome? Anatol J Cardiol 2015; 15: 385-90.

[26] Acet H, Ertaş F, Akıl MA et al. Novel predictors of infarctrelated artery patency for ST-segment elevation myocardial infarction: Platelet-to-lymphocyte ratio, uric acid, and neutrophil-to-lymphocyte ratio. Anatol J Cardiol 2015; 15: 648-56. DOI: 10.5152/akd.2014.5592.

[27] Yayla C, Akbog a MA, Canpolat U et al. Platelet to Lymphocyte Ratio Canbe a Predictor of Infarct-Related Artery Patency in Patients with ST-Segment Elevation Myocardial Infarction. Angiology. 2015 Oct; 66 (9): 831-6. doi: 10.1177/0003319715573658. 